\title{
Long-term impact of dry eye symptoms on vision-related quality of life after phacoemulsification surgery
}

\author{
Wenwen Xue $\cdot$ Ming-ming Zhu $\cdot$ Bi-jun Zhu $\cdot$ Jian-nan Huang $\cdot$ \\ Qian Sun · Yu-yu Miao $\cdot$ Hai-dong Zou
}

Received: 13 July 2017/ Accepted: 20 January 2018/Published online: 1 February 2018

(C) The Author(s) 2018. This article is an open access publication

\begin{abstract}
Purpose To observe the long-term changes in dry eye symptoms and vision-related quality of life in agerelated cataract patients after phacoemulsification.

Methods A total of 101 cataract patients after phacoemulsification combined with IOL implantation (Ph-IOL) in one eye were enrolled. Visual acuity, tear film breakup time (BUT), and Schirmer test 1 (ST1) were measured before and 1, 3, and 6 months after surgery. Ocular Surface Disease Index (OSDI) scores
\end{abstract}

Wenwen Xue and Ming-ming Zhu have contributed equally to this work.

W. Xue $\cdot$ M. Zhu $\cdot$ B. Zhu $\cdot$ J. Huang .

Q. Sun $\cdot$ Y. Miao $\cdot$ H. Zou

Department of Ophthalmology, Shanghai General

Hospital, Shanghai Jiao Tong University School of

Medicine, Shanghai, China

W. Xue $\cdot$ M. Zhu $\cdot$ B. Zhu $\cdot$ J. Huang .

Q. Sun · Y. Miao · H. Zou

Shanghai Key Laboratory of Ocular Fundus Diseases,

Shanghai, China

W. Xue $\cdot$ M. Zhu $\cdot$ B. Zhu $\cdot$ J. Huang

Q. Sun $\cdot$ Y. Miao $\cdot$ H. Zou $(\bowtie)$

Shanghai Engineering Center for Visual Science and

Photomedicine, Shanghai, China

e-mail: zouhaidong@263.net

W. Xue $\cdot$ M. Zhu $\cdot$ J. Huang $\cdot$ H. Zou

Department of Preventative Ophthalmology, Shanghai

Eye Diseases Prevention and Treatment Center, No. 380,

Kangding Road, Jingan District, Shanghai 200040, China were used to evaluate the severity of dry eye symptoms. Utility values were assessed by the time trade-off (TTO), standard gamble for death (SGD), standard gamble for blindness (SGB) and rating scale (RS).

Results The average LogMAR visual acuity in the operated eye was $1.35 \pm 0.50$ and increased rapidly after $\mathrm{Ph}$-IOL, approaching a peak at 3 months $(0.26 \pm 0.15)$. The BUT and ST1 results decreased abruptly 1 month after surgery and gradually recovered until 6 months. OSDI scores increased significantly after surgery and gradually decreased until 6 months. Utility values evaluated by TTO, SGD, SGB and RS before surgery were $0.67 \pm 0.19$, $0.75 \pm 0.15,0.67 \pm 0.20$ and $0.2 \pm 0.18$, respectively, and increased to $0.91 \pm 0.06,0.98 \pm 0.04$, $0.92 \pm 0.52$ and $0.91 \pm 0.06,6$ months after. Utility values measured with TTO, SGB or RS correlated significantly $(P<0.05)$ with visual acuity and OSDI scores pre- and postoperatively.

Conclusions Dry eye symptoms persist more than 3 months after Ph-IOL. Utility values were negatively influenced by dry eye symptoms.

Keywords Cataract - Phacoemulsification · Dry eye $\cdot$ Utility $\cdot$ Visual acuity 


\section{Introduction}

Age-related cataract is still the leading cause of blindness in most developing countries, and as populations age, the number of cataract patients will be constantly rising. Cataract surgery can improve the visual acuity largely. However, a significant number of patients complain of postoperative dry eye symptoms such as dry, foreign body sensation and shorttime but frequent blurred vision [1-5]. From our experience, the patients may blame the surgery (and their surgeon) for the apparently new development of dry eye symptoms and generally do not realize that their problem stems from ocular surface disease rather than the surgical procedure. To avoid these accusations and to inform patients preoperatively of the possibility of dry eye symptoms following surgery, it is equally important to provide information about when these symptoms will diminish or disappear. The severity of dry eye symptoms has been reported to peak 7 days after cataract surgery and to persist for 3 months, and it can become both physically and psychologically debilitating $[1,2]$.

Patient subjective preferences appear to be more sensitive indicators of dry eye symptoms when compared to traditional biomedical parameters such as visual acuity and contrast sensitivity. The OSDI is a validated, reliable questionnaire that assesses a patient's subjective preferences with dry eye disease, including those that occur following cataract surgery $[1,2,6]$. The OSDI is now perhaps the most commonly used dry eye-specific questionnaire due to its convenience in clinical settings [2]. However, the limitations of OSDI, which also exist in other item-based questionnaires, are inevitable. For example, no itembased questionnaire can address every aspect of a given condition and how it affects VRQoL such that investigators are able to incorporate the data into economic analyses.

Utility value, which originated in medical economics [7], is another alternative for evaluating quality of life. Several researchers consider utility value to be among the most all-encompassing qualityof-life instruments that can be easily used to compare the impact on quality of life of different ophthalmic conditions [8, 9]. Based on different building concepts, there are three methods to assess utility value: TTO, SG and RS. TTO is the most commonly used method. Studies that have used TTO to measure utility value associated with dry eye disease have found that severe dry eye utilities were similar to those reported for dialysis and severe angina $[10,11]$. To date, there has been no report demonstrating the utility value change associated with dry eye symptoms after cataract surgery. Furthermore, there has been no discussion regarding which utility value assessing method is proper to evaluate quality-of-life changes due to dry eye symptoms after cataract surgery.

In the present study, we report preoperative and 1-month, 3-month and 6-month postoperative visual acuity, BUT, ST1 result, OSDI scores and utility values among patients who underwent Ph-IOL. The utility values for the TTO, SG and RS methods are all presented. We consider that the study results reflect the long-term impact of dry eye symptoms on VRQoL among patients after Ph-IOL surgery.

\section{Methods}

A prospective hospital-based study was performed with eligible patients who received uneventful Ph-IOL in one eye in the Department of Ophthalmology, Shanghai General Hospital, between January 2014 and June 2015. The patients who understood and agreed to cooperate by attending regular follow-up visits within 6 months after surgery were included in this study. Exclusion criteria were as follows: (1) limited ability to communicate effectively with the investigator and inability to complete the questionnaires with the investigator's assistance; (2) presence of other eye diseases before and after surgery, such as ocular trauma, keratopathy, glaucoma, dacrycystitis, uveitis, retinal detachment and age-related macular degeneration; (3) the presence of other systemic acute or chronic diseases, such as diabetes and coronary heart disease; (4) intraoperative or postoperative complications of $\mathrm{Ph}-\mathrm{IOL}$ such as posterior capsule rupture and pseudophakic retinal detachment; (5) the use of artificial tears or other topical ophthalmologic agents for more than 2 weeks during the study period that potentially impacted the efficacy assessment of the study; (6) apparent dry eye symptoms or tear film BUT less than $5 \mathrm{~s}$ or ST1 result less than $5 \mathrm{~mm}$ before cataract surgery and (7) history of allergic conjunctivitis.

All patients received $\mathrm{Ph}-\mathrm{IOL}$ procedure through clear corneal incisions. The patients enrolled all 
received retrobulbar anesthesia. Within 2 weeks after surgery, Tobradex $(0.3 \%$ tobramycin and $1 \%$ dexamethasone, Alcon, Fort Worth, TX) eyedrops were applied four times a day, a non-steroidal anti-inflammatory eyedrops were applied four times a day, and tropicamide was applied once every evening before bed.

Basic information concerning the enrolled patients including gender, age, job occupation and education level was collected. A subjective questionnaire was completed prior to the objective examination that was administered in the following order: OSDI, utility value assessment, visual acuity check, slit lamp examinations, BUT and ST1 tests. All of the questionnaires and examinations were administered in the morning. Two well-trained investigators completed the objective examination and assisted the patients in finishing the subjective questionnaires with face-toface communication. An ophthalmologist with sufficient experience in ophthalmologic examination, OSDI and utility study supervised the entire project.

The Snellen chart was used to examine the patients' bilateral distance presenting visual acuity before and after surgery. We did not use best-corrected vision acuity (BCVA) in this study; as many senior citizens in China might have a reduced desire for fine work, they are satisfied with presenting vision acuity and are not willing to wear spectacles. Visual acuity of the eyes was recorded and transformed into LogMAR visual acuity. Weighted average LogMAR represents a summary score of visual acuity encompassing visual information from both eyes, with the better eye given a weight of 0.75 and the worse eye given a weight of 0.25 . This quantification of visual function weights equally changes in the resolvable visual angle across the visual spectrum [12]. Slit lamp examinations evaluated the eyelids, conjunctiva, cornea, iris and chamber for inflammation or structural change. Specific ocular surface examination and grading methods for dry eye symptoms were as follows: (1) BUT was measured in second(s) using fluorescein. Measurements were repeated three times, and the mean value was calculated; (2) ST1 was measured using a piece of standard $5 \mathrm{~mm} \times 35 \mathrm{~mm}$ filter paper without surface anesthesia.

The severity of dry eye symptoms was evaluated using the OSDI questionnaire. The OSDI, developed by the Outcomes Research Group at Allergan Inc (Irvine, Calif), is a 12-item questionnaire designed to evaluate ocular symptoms consistent with dry eye disease and their impact on vision-related functioning $[6,13]$. The higher the score, the more severe the symptoms. (OSDI score $=$ Total Sum of Answers $\times 25 /$ Number of Questions Answers; in this case, OSDI score $=$ total sum of answers $\times 25 / 6$.) For the purpose of the current study, only six items were used as they were considered not to be affected by visual acuity change (Table 1). These items are graded on a scale from 0 to 4 , where 0 indicates none of the time; 1 , some of the time; 2 , half of the time; 3 , most of the time; 4, all of the time. Questions on blurred vision, poor vision and visual performance were omitted so that any change in post-op scores would be related purely to symptoms and not affected by improved vision.

The traditional TTO, SG and RS methods were used to evaluate utility values. TTO requires the patient to estimate their own expected remaining life and then choose $X$ years that they wish to live with their present visual status or with perfect visual status at the cost of reduction of $t$ years from the expected life span. Then, the utility value is calculated as $\mathrm{U}_{\text {TTO }}=1-t / X$. SG based on the highest risk $Y(\%)$ for the maximum treatment failure: death (SGD) or blindness (SGB) that the patient would be willing to accept. $U_{\mathrm{SG}}=1-Y$. In the RS approach, patients are asked to mark the position $(P)$ on a graduated ruler (0-100 points) that could best represent the present visual status, and $U_{\mathrm{RS}}=1-P \%$. Utility values range from 1.0 (perfect visual status) to 0.0 (blindness or death) $[10,14]$.

SPSS17.0 software was used for statistical analysis. Statistical analytic methods included Spearman correlation analysis, parametric paired $t-t$ test and nonparametric Wilcoxon signed rank test. $p<0.05$ indicated a statistically significant difference.

This study was approved by the Ethics Committee of Shanghai General Hospital, Shanghai Jiao Tong University, in accordance with the tenets of the Declaration of Helsinki. Written informed consent was obtained from each participant.

\section{Results}

A total of 101 patients with age-related cataracts (101 eyes) were enrolled in this study. Patient characteristics are presented in Table 2. A vast majority of the 
Table 1 Items chose from OSDI

\begin{tabular}{l} 
Item \\
\hline Have you experienced any of the following during the last week? \\
Eyes that are sensitive to light \\
Eyes that feel gritty
\end{tabular}

Table 2 Patient characteristics

\begin{tabular}{lll}
\hline Category & Group & \\
\hline Gender, $N(\%)$ & Male & $47(46.53 \%)$ \\
& Female & $54(53.47 \%)$ \\
Age (year) & Range & $43-84$ \\
Education year, $N(\%)$ & Mean (SD) & $66.33(9.69)$ \\
& $0-6$ & $17(16.83 \%)$ \\
& $7-12$ & $22(21.78 \%)$ \\
Working status, $N(\%)$ & $>12$ & $62(61.39 \%)$ \\
& Retired & $71(70.30 \%)$ \\
& In service & $30(29.70 \%)$ \\
\hline
\end{tabular}

patients were older than 50 years (99 patients, 98.02\%).

All 101 patients received cataract surgery in one eye. Preoperative and postoperative visual acuity and ocular surface examination results of the patients are shown in Table 3. The visual acuity in the operated eye increased rapidly 1 month after cataract surgery and steadily approached a peak at 3 months, with no further change at 6 months. Because the visual acuity of most of the fellow eyes did not change during the 6-month observation time, the weighted average visual acuity showed a similar changing curve after surgery. Conversely, the BUT and ST1 results decreased abruptly 1 month after surgery and then gradually recovered. At the 3-month postoperative time point, the BUT and ST1 results were still lower than the preoperative level, but they kept rising and finally approached the preoperative level at 6 months. However, there were still significant differences between the preoperative and 6-months postoperative BUT (paired test $t=7.59, p<0.01$ ) or ST1 (paired test $t=8.64, p<0.01)$. As shown in Table 4, the
OSDI scores increased significantly after surgery and then gradually decreased by 6 months. At the end of 6 months, the OSDI scores were still higher than the preoperative scores (Wilcoxon signed rank test $Z=3.11, p<0.01)$.

Preoperative and postoperative utility values of the 101 patients are shown in Table 5. After cataract surgery, all of the utility values increased steadily until 3 months, irrespective of the method of assessment. Among them, SGD resulted in the highest utility value, and RS resulted in the lowest. During the 3-6 months postoperative time period, the TTO, SGB and RS utility values continued to increase, while the SGD remained stable.

As shown in Table 6, the utility values measured with TTO, SGD, SGB and RS did not correlate with gender, age, educational level, BUT or ST1. There were significant correlations between the preoperative and postoperative weighted average visual acuity and utility values measured with TTO, SGB or RS, except between the visual acuity of the surgery eye and RS result 6 months postoperatively. These utility values also significantly correlated with the OSDI scores postoperatively, although the correlation coefficients were lower than those between the utility values and weighted average visual acuity. The utility values measured by SGD correlated with the preoperative average visual acuity or OSDI scores 1 month after surgery, but not with visual acuity or OSDI scores 3 or 6 months postoperatively.

As shown in Table 7, there was a significant difference between any two of the utility values assessed by TTO, SG and RS before cataract surgery. After cataract surgery, except for utility values assessed by TTO and SGB, most of the pairwise comparison of other utility values indicated significant differences. The preoperative and 1-month, 3-months 
Table 3 Preoperative and postoperative visual acuity and specific ocular surface examination results: values were shown as mean (SD)

\begin{tabular}{lllll}
\hline & Preoperative & 1 month postoperative & 3 months postoperative & 6 months postoperative \\
\hline LogMAR visual acuity surgery eye & $1.35(0.50)$ & $0.30(0.18)$ & $0.26(0.15)$ & $0.26(0.14)$ \\
& & $20.46^{\dagger *}$ & $5.91^{\dagger * *}$ & $0.03,0.91^{* *}$ \\
Weighted LogMAR visual acuity & $0.58(0.17)$ & $0.26(0.12)$ & $0.24(0.11)$ & $0.24(0.10)$ \\
& & $19.57^{\dagger *}$ & $5.61^{\dagger * *}$ & $0.37,0.71 * * *$ \\
Tear film breakup time & \multirow{2}{*}{$9.10(3.77)$} & $4.44(3.32)$ & $7.29(3.48)$ & $8.64(3.82)$ \\
Schirmer test 1 & & $9.28^{\dagger *}$ & $21.86^{\dagger * *}$ & $2.61,0.01^{* * *}$ \\
& \multirow{2}{*}{$12.90(8.31)$} & $6.06(3.12)$ & $8.75(4.44)$ & $11.99(8.47)$ \\
& & $12.70^{\dagger *}$ & $5.85^{\dagger * *}$ & $3.90^{\dagger * * *}$
\end{tabular}

Paired $t-t$ test was performed

*Comparing preoperative and 1-month postoperative results

**Comparing 1- and 3-month postoperative results

***Comparing 3- and 6-month postoperative results

${ }^{\dagger} p<0.01$

${ }^{\ddagger} p<0.05$

Table 4 Preoperative and postoperative OSDI 6 items and total scores: values were shown as median (range)

\begin{tabular}{|c|c|c|c|c|}
\hline & Preoperative & $\begin{array}{l}1 \text { month } \\
\text { postoperative }\end{array}$ & $\begin{array}{l}3 \text { months } \\
\text { postoperative }\end{array}$ & $\begin{array}{l}6 \text { months } \\
\text { postoperative }\end{array}$ \\
\hline Item "sensitive to light" & $1(0-1)$ & $\begin{array}{l}2(0-4) \\
7.58^{\dagger *}\end{array}$ & $\begin{array}{l}1(0-2) \\
6.41^{\dagger * *}\end{array}$ & $\begin{array}{l}1(0-1) \\
4.56^{\dagger * * * *}\end{array}$ \\
\hline Item "feel gritty" & $0(0-1)$ & $\begin{array}{l}2(0-4) \\
8.08^{\dagger *}\end{array}$ & $\begin{array}{l}1(0-2) \\
5.54^{\dagger * *}\end{array}$ & $\begin{array}{l}1(0-2) \\
6.80^{\dagger * * * *}\end{array}$ \\
\hline Item "painful or sore eyes" & $0(0-1)$ & $\begin{array}{l}2(0-4) \\
8.22^{\dagger *}\end{array}$ & $\begin{array}{l}2(0-2) \\
4.97^{\dagger * *}\end{array}$ & $\begin{array}{l}1(0-2) \\
7.15 * * *\end{array}$ \\
\hline Item "windy conditions" & $0(0-1)$ & $\begin{array}{l}2(0-4) \\
8.17^{\dagger *}\end{array}$ & $\begin{array}{l}1(0-2) \\
6.16^{\dagger * *}\end{array}$ & $\begin{array}{l}1(0-1) \\
7.10 * * *\end{array}$ \\
\hline Item "places or areas with low humidity" & $0(0-1)$ & $\begin{array}{l}2(0-4) \\
8.23^{\dagger *}\end{array}$ & $\begin{array}{l}2(0-2) \\
4.89^{\dagger * *}\end{array}$ & $\begin{array}{l}1(0-1) \\
7.55 * * *\end{array}$ \\
\hline Item "areas that are air-conditioned" & $1(0-1)$ & $\begin{array}{l}2(0-4) \\
7.95^{\dagger *}\end{array}$ & $\begin{array}{l}2(0-2) \\
5.31^{\dagger * *}\end{array}$ & $\begin{array}{l}1(0-1) \\
7.24^{\dagger * * * *}\end{array}$ \\
\hline OSDI score & $12.5(0-25)$ & $\begin{array}{l}58.33(29.17-83.33) \\
8.726^{\dagger *}\end{array}$ & $\begin{array}{l}37.5(16.67-54.17) \\
8.318^{\dagger * *}\end{array}$ & $\begin{array}{l}12.5(0-29.17) \\
8.708^{\dagger * * *}\end{array}$ \\
\hline
\end{tabular}

Wilcoxon signed rank test $Z$ was performed

*Comparing preoperative and 1-month postoperative results

**Comparing 1- and 3-month postoperative results

$* * *$ Comparing 3- and 6-month postoperative results

${ }^{\dagger} p<0.01$

and 6-months postoperative LogMAR visual acuity, BUT, ST1, OSDI scores and utility values assessed by the TTO, SG and RS, among 101 patients who underwent $\mathrm{Ph}-\mathrm{IOL}$, are shown in Fig. 1.
In the present study, the correlation coefficient between OSDI scores and the utility value assessed by RS was much higher than that of TTO or SG at the 6-month postoperative time point. We therefore 
Table 5 Preoperative and postoperative utility values

\begin{tabular}{|c|c|c|c|c|}
\hline & Preoperative & 1 month postoperative & 3 months postoperative & 6 months postoperative \\
\hline \multicolumn{5}{|l|}{ Time trade-off } \\
\hline Mean (SD) & $0.67(0.19)$ & $0.78(0.10)$ & $0.85(0.09)$ & $0.91(0.06)$ \\
\hline Paired $t$ test, $p$ & & $6.21^{\dagger *}$ & $12.80^{\dagger * *}$ & $11.27^{\dagger * * *}$ \\
\hline \multicolumn{5}{|c|}{ Standard gamble for death } \\
\hline Mean (SD) & $0.75(0.15)$ & $0.93(0.09)$ & $0.98(0.06)$ & $0.98(0.04)$ \\
\hline Paired $t$ test, $p$ & & $13.40^{\dagger *}$ & $5.28^{\dagger * *}$ & $1.16,0.25^{* * *}$ \\
\hline \multicolumn{5}{|c|}{ Standard gamble for blindness } \\
\hline Mean (SD) & $0.67(0.20)$ & $0.78(0.10)$ & $0.86(0.09)$ & $0.92(0.52)$ \\
\hline Paired $t$ test, $p$ & & $5.87^{\dagger *}$ & $12.83^{\dagger * *}$ & $9.10^{\dagger * * *}$ \\
\hline \multicolumn{5}{|l|}{ Rating scale } \\
\hline Mean (SD) & $0.72(0.18)$ & $0.81(0.11)$ & $0.86(0.10)$ & $0.91(0.06)$ \\
\hline Paired $t$ test, $p$ & & $5.86^{\dagger *}$ & $8.63^{\dagger * *}$ & $5.11^{\dagger * *}$ \\
\hline
\end{tabular}

*Comparing preoperative and 1-month postoperative results

**Comparing 1- and 3-month postoperative results

***Comparing 3- and 6-month postoperative results

${ }^{\dagger} p<0.01$

speculate that RS had a higher sensitivity for dry eye symptoms with a relatively mild influence on quality of life. Then, the regression analysis was performed based on the utility values 6 months postoperatively; $\mathrm{RS}=1.007-0.028$ OSDI scores $\quad(F=153.349$, $\left.p<0.01 ; R^{2}=0.608\right)$.

\section{Discussion}

Traditionally, the judgment of a "satisfactory" cataract surgery has been based on biomedical indicators such as visual acuity. However, maintaining a healthy ocular surface is essential for achieving the best visual outcome in cataract patients. Ocular surface preparation is beneficial not only in patients with established ocular surface disease, but also in those with minimal signs or symptoms of surface disease [15]. Increasingly, attention is being devoted to measuring VRQoL, and interest in outcomes research has grown from a recognition that patients are more interested in how interventions affect their well-being than in improvements in visual acuity [16]. The good news is that many studies have confirmed that VRQoL of patients with cataracts significantly improved after Ph-IOL [17-21]. In the present study, we also confirmed that after Ph-IOL, both visual acuity and utility value increased significantly. However, these indicators did not increase in parallel, especially in the 3-6 months postoperative time frame. Furthermore, utility values assessed by TTO, SGB and RS continued to increase, while visual acuity remained stable. The cause of this lack of uniformity is not entirely clear, though we speculate that it is in part a result of dry eye symptoms.

Dry eye symptoms may be aggravated after cataract surgery. Some studies ascribed these symptoms to deterioration in corneal sensitivity and tear physiology [22]. Other studies reported serious squamous metaplasia or mean goblet cell density decreases in the epithelial layer of the globe conjunctiva $[1,5]$. Several studies confirmed that BUT, ST1 and OSDI scores and quality of life did not recover to the preoperative level by 3 months postoperatively $[1,2]$. No report has demonstrated related quality-of-life change more than 3 months postoperatively. In the present study, we also found that although the dry eye symptoms evaluated by OSDI items did not resolve 3 months after surgery, they ultimately subsided 6 months postoperatively. In the 3 and 6 months postoperative time period, utility values assessed by TTO, SGB and RS all correlated significantly with dry eye symptoms. 
Table 6 Spearman correlation coefficients between utility values and gender, age, education level, tear film breakup time, Schirmer test 1, OSDI item scores, visual acuity before and after cataract surgery
${ }^{\dagger} p<0.01$

${ }^{\ddagger} p<0.05$

\begin{tabular}{|c|c|c|c|c|}
\hline & TTO & SGD & SGB & $\mathrm{RS}$ \\
\hline \multicolumn{5}{|l|}{ Before cataract surgery } \\
\hline Gender & 0.09 & 0.06 & 0.07 & 0.13 \\
\hline Age & 0.17 & 0.19 & 0.19 & 0.15 \\
\hline Education level & -0.12 & -0.12 & -0.13 & -0.06 \\
\hline LogMAR visual acuity of the surgery eye & -0.04 & 0.01 & -0.04 & 0.01 \\
\hline Weighted average LogMAR visual acuity & $-0.49^{\dagger}$ & $-0.64^{\dagger}$ & $-0.47^{\dagger}$ & $-0.65^{\dagger}$ \\
\hline Tear film breakup time, & 0.11 & -0.02 & -0.12 & 0.01 \\
\hline Schirmer test 1 & $-0.28^{\dagger}$ & -0.19 & $-0.30^{\dagger}$ & -0.18 \\
\hline OSDI score & -0.03 & -0.06 & 0.01 & -0.06 \\
\hline \multicolumn{5}{|l|}{ 1-month after cataract surgery } \\
\hline Gender & -0.06 & -0.15 & -0.06 & -0.07 \\
\hline Age & 0.15 & 0.19 & 0.16 & 0.19 \\
\hline Education level & -0.14 & -0.03 & -0.13 & -0.06 \\
\hline LogMAR visual acuity of the surgery eye & $-0.60^{\dagger}$ & $-0.41^{\dagger}$ & $-0.58^{\dagger}$ & $-0.60^{\dagger}$ \\
\hline Weighted average LogMAR visual acuity & $-0.82^{\dagger}$ & $-0.56^{\dagger}$ & $-0.82^{\dagger}$ & $-0.85^{\dagger}$ \\
\hline Tear film breakup time, & -0.15 & -0.15 & -0.17 & -0.13 \\
\hline Schirmer test 1 & -0.15 & -0.15 & -0.17 & -0.13 \\
\hline OSDI score & $-0.71^{\dagger}$ & $-0.24^{*}$ & $-0.71^{\dagger}$ & $-0.71^{\dagger}$ \\
\hline \multicolumn{5}{|l|}{3 months after cataract surgery } \\
\hline Gender & -0.06 & -0.06 & -0.01 & -0.07 \\
\hline Age & 0.17 & 0.13 & 0.17 & 0.16 \\
\hline Education level & -0.13 & -0.03 & -0.02 & -0.05 \\
\hline LogMAR visual acuity of the surgery eye & $-0.73^{\dagger}$ & -0.06 & $-0.68^{\dagger}$ & $-0.71^{\dagger}$ \\
\hline Weighted average LogMAR visual acuity & $-0.99^{\dagger}$ & 0.02 & $-0.95^{\dagger}$ & $-0.98^{\dagger}$ \\
\hline Tear film breakup time & 0.06 & -0.08 & -0.01 & 0.07 \\
\hline Schirmer test 1 & -0.07 & -0.01 & -0.07 & -0.06 \\
\hline OSDI score & $-0.84^{\dagger}$ & 0.14 & $-0.83^{\dagger}$ & $-0.84^{\dagger}$ \\
\hline \multicolumn{5}{|l|}{6 months after cataract surgery } \\
\hline Gender & -0.03 & 0.01 & -0.05 & -0.02 \\
\hline Age & 0.14 & 0.16 & 0.13 & 0.08 \\
\hline Education level & -0.12 & -0.02 & -0.10 & -0.04 \\
\hline LogMAR visual acuity of the surgery eye & $-0.58^{\dagger}$ & -0.01 & $-0.53^{\dagger}$ & -0.15 \\
\hline Weighted average LogMAR visual acuity & $-0.82^{\dagger}$ & 0.01 & $-0.78^{\dagger}$ & $-0.88^{\dagger}$ \\
\hline Tear film breakup time & 0.02 & -0.01 & -0.04 & 0.07 \\
\hline Schirmer test 1 & -0.10 & 0.03 & -0.11 & -0.06 \\
\hline OSDI score & $-0.56^{\dagger}$ & 0.02 & $-0.44^{\dagger}$ & $-0.80^{\dagger}$ \\
\hline
\end{tabular}

Therefore, it is suggested that the long-term persistent dry eye symptoms affected health-related quality-oflife recovery until the 6-month time point.

We found it difficult to separate the influence of visual acuity from dry eye symptoms on quality of life with the utility values obtained in the present study. The correlation coefficients between the utility values and weighted average LogMAR visual acuity were all higher than those between utility values and OSDI scores, demonstrating that though dry eye symptoms had some effect, visual acuity was still the most direct and most important factor influencing quality of life after cataract surgery. To more precisely ascertain the influence of dry eye symptoms on patients' quality of life after $\mathrm{Ph}-\mathrm{IOL}$, it is necessary to focus on utility values in patients with different levels of visual acuity 
Table 7 Pairwise comparison of utility values measured with TTO, SGD, SGB and RS before and after cataract surgery (paired $t$ test: $t$ value)

\begin{tabular}{lllllll}
\hline & TTO-SGD & TTO-SGB & TTO-RS & SGD-SGB & RS-SGD & RS-SGB \\
\hline Before cataract surgery & $7.17^{\dagger}$ & $1.69 \dagger$ & $4.74^{\dagger}$ & $6.35^{\dagger}$ & $4.60^{\dagger}$ & $4.11^{\dagger}$ \\
1 month after cataract surgery & $16.35^{\dagger}$ & 0.38 & $8.08^{\dagger}$ & $14.93^{\dagger}$ & $13.73^{\dagger}$ & $5.88^{\dagger}$ \\
3 months after cataract surgery & $11.83^{\dagger}$ & 0.36 & $2.40^{\dagger}$ & $11.23^{\dagger}$ & $11.10^{\dagger}$ & 1.49 \\
6 months after cataract surgery & $9.60^{\dagger}$ & 1.23 & 1.28 & $9.75^{\dagger}$ & $11.00^{\dagger}$ & $2.17^{\ddagger}$ \\
\hline
\end{tabular}

${ }^{\dagger} p<0.01$

${ }^{\ddagger} p<0.05$
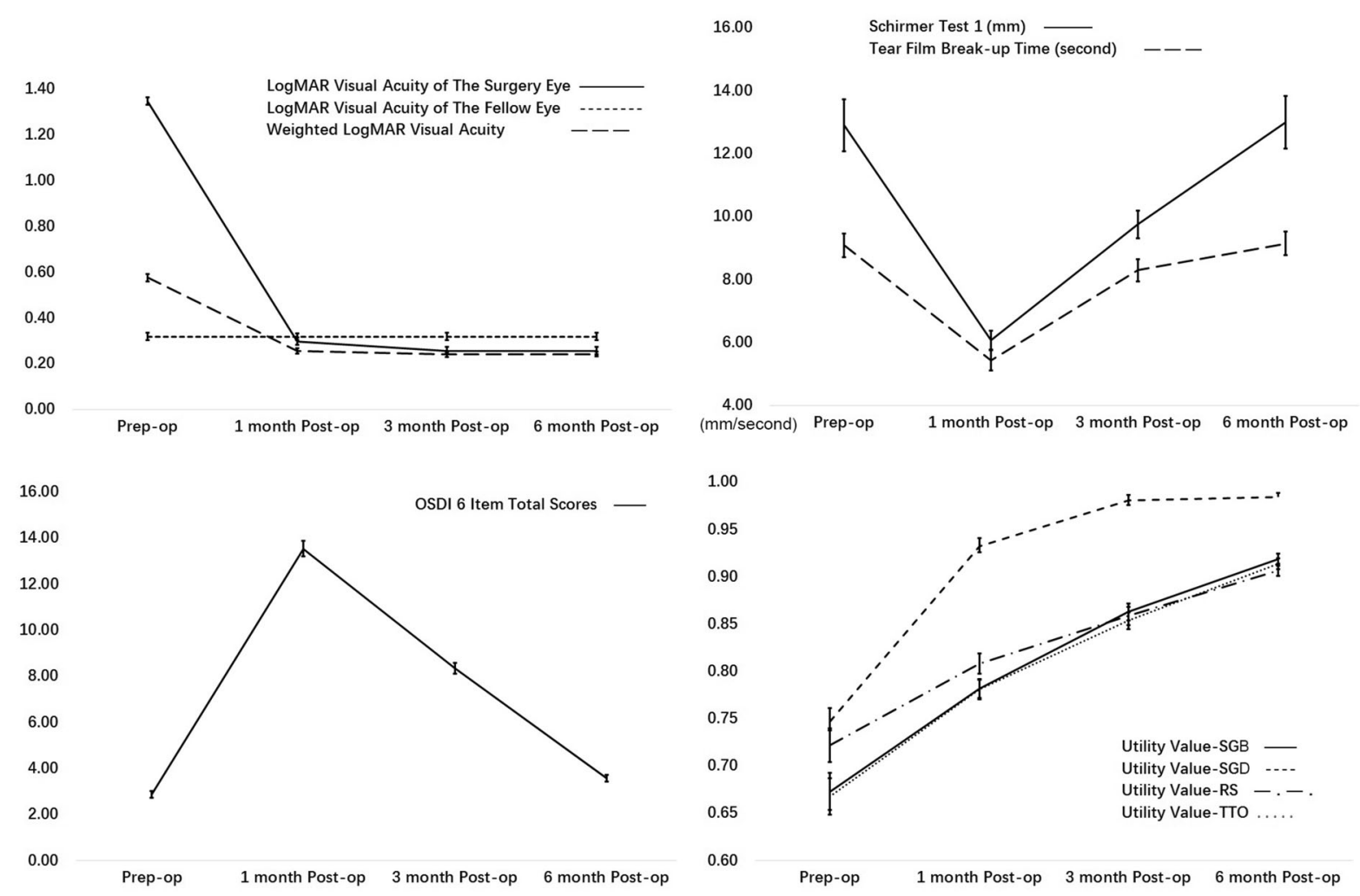

Fig. 1 Preoperative and 1-, 3- and 6-month postoperative values for a LogMAR visual acuity, b Schirmer test 1 and tear film breakup time, c OSDI 6 item scores and $\mathbf{d}$ utility values

or dry eye symptoms. Because most of the eyes achieved good visual acuity $(\geq 20 / 30)$ after surgery and most of the patients had slight to mild dry eye symptoms, we were unable to divide the patients into different subgroups according to their visual acuity or dry eye symptoms. Future studies on different groups may help to substantiate the hypothesis.

Patient subjective feelings are never synchronous with objective examination results. In present study, no significant correlation was found between OSDI scores and BUT or ST1, which was consistent with other studies. For example, Schiffman and associates [6] reported that no significant correlation between the OSDI and the clinical objective examinations such as BUT, ST1 and fluorescein staining. Indeed, the regression equation indicated that OSDI was a significant predictor, which suggested higher OSDI scores may have negative effects on the utility value. 
However, quality of life is an individual experience that is influenced by different cultural and value systems [26]. Sensory, cognitive, physical and mental status change drastically after cataract surgery. It is uneasy to say OSDI significantly influenced patients' quality of life. Statistical significance may not reflect the whole picture of the "clinical significance" in this change or difference. Educational level and family income are also sometime related to utility values [27]. In the present study, data were obtained through faceto-face interviews, and the influence of education level on utility might be somewhat weaker. No significant correlation between educational level and utility was observed. Populations with different income levels also have different requirements for supporting quality of life. The majority of enrolled patients were retirees. According to Shanghai's policy, their incomes were almost equivalent, and therefore, "family income" was not included in the analysis.

At 6 months postoperatively, utility values obtained with TTO, SG and RS were all higher than or equal to 0.91 , suggesting that patients' overall postoperative visual status could basically meet their daily needs. Many researchers have assessed the consistency of utility values by different multidimensional utility indicators and found that different methods usually led to different results [23-25]. Here, we used TTO, SG and RS to evaluate the quality of life of patients who underwent cataract surgery and also found different results, as shown in Table 7. These differences are mainly due to the different assessing principles that underlie the different methods. There is a gamble concept in TTO and SG, i.e., the prerequisite for the patients to obtain a higher quality of life is for them to give up something, such as life and visual acuity. To some extent, it also reflects the patients' fear of blindness or death. Time trade-off takes reduction in the expected life span as the cost, while SG takes the risk of immediate death (SGD) or immediate blindness (SGB) in exchange for perfect visual status. Therefore, it is plausible that the utility values of TTO and SGB were similar when visual acuity became better after cataract surgery. However, because it was clearly unacceptable to risk immediate death in exchange for better visual status when the patient's visual quality could meet daily needs, the utility values assessed by SGD were much higher than other utility values after surgery. Rating scale is simply an assessment of present visual status and reflects the individual's level of satisfaction. Overall, because utility values assessed by TTO, SGB and RS all correlated strongly with dry eye symptoms between 1 and 6 months postoperatively, we consider that these three utility value assessing methods are all proper for evaluating quality-of-life changes due to dry eye symptoms after cataract surgery. In the present study, the correlation coefficient between OSDI scores and the utility value assessed by RS was much higher than that of TTO or SG at the 6 months postoperative time point. We therefore speculate that RS had a higher sensitivity for dry eye symptoms with a relatively mild influence on quality of life.

The limitations of the present study should not be omitted. First, it is difficult to eliminate interference from artificial tears on ophthalmologic examination on dry eye symptoms. We did not include patients with severe dry eye symptoms after surgery. The reason is that long-term usage of artificial tears or other eye solutions were obligatory treatments that may interfere with the natural recovery history of dry eye symptoms and further influence the quality-of-life change due to dry eye symptoms. Second, steroids such as dexamethasone were used in the first 2 weeks postoperatively and were a regular prescription drug of the post-surgical therapy. Because steroids reduce inflammation in the injured ocular surface epithelium, they may interfere with dry eye symptoms and related quality of life in the first month, though this did not extend to 3 months postoperatively. Third, the present study did not include corneal findings (staining score) and may indicate a need for further investigation.

In conclusion, dry eye symptoms are common after Ph-IOL surgery, persist for more than 3 months and subside 6 months postoperatively. The healthy utility values were negatively influenced by dry eye symptoms and reached a peak at 6 months. Therefore, it is strongly suggested that in the preoperative period, surgeons and the staff inform the patients about dry eye symptoms such as dry, foreign body sensation and short-time but frequent blurred vision. This information will help the patients separate these symptoms from those related to the cataract surgery, thus decreasing the likelihood of misplaced accusations. After surgery, the surgeons and staff should pay more attention to the ocular surface changes and aggressively treat the dry eye symptoms. Simultaneously, patients should be encouraged by the knowledge that most dry eye symptoms diminish after 6 months. 
These recommendations will likely provide additional benefits beyond the improvement in visual acuity.

Authors' contributions WX, MZ and $\mathrm{HZ}$ wrote this manuscript. BZ, JH, QS and YM researched the data and contributed to writing the manuscript. All authors read and approved the final manuscript.

\section{Compliance with ethical standards}

Conflict of interest No conflicting relationship exists for any author.

Informed consent Informed consent was obtained from all individual participants included in the study.

Ethical approval All procedures performed in studies involving human participants were in accordance with the ethical standards of the institutional and/or national research committee and with the 1964 Declaration of Helsinki and its later amendments or comparable ethical standards. The institutional review board of the Shanghai General Hospital, Shanghai Jiao Tong University School of Medicine, Shanghai, China, approved the study. The registration number of accepted IRB was 2013KY081.

Open Access This article is distributed under the terms of the Creative Commons Attribution 4.0 International License (http:// creativecommons.org/licenses/by/4.0/), which permits unrestricted use, distribution, and reproduction in any medium, provided you give appropriate credit to the original author(s) and the source, provide a link to the Creative Commons license, and indicate if changes were made.

\section{References}

1. Li XM, Hu L, Hu J, Wang W (2007) Investigation of dry eye disease and analysis of the pathogenic factors in patients after cataract surgery. Cornea 26(9 Suppl 1):S16-S20

2. Kasetsuwan N, Satitpitakul V, Changul T, Jariyakosol S (2013) Incidence and pattern of dry eye after cataract surgery. PLoS ONE 8(11):e78657

3. Cho YK, Kim MS (2009) Dry eye after cataract surgery and associated intraoperative risk factors. Korean J Ophthalmol 23(2):65-73

4. Sitompul R, Sancoyo GS, Hutauruk JA, Gondhowiardjo TD (2008) Sensitivity change in cornea and tear layer due to incision difference on cataract surgery with either manual small-incision cataract surgery or phacoemulsification. Cornea 27(Suppl 1):S13-S18

5. Oh T, Jung Y, Chang D, Kim J, Kim H (2012) Changes in the tear film and ocular surface after cataract surgery. Jpn J Ophthalmol 56(2):113-118

6. Schiffman RM, Christianson MD, Jacobsen G, Hirsch JD, Reis BL (2000) Reliability and validity of the ocular surface disease index. Arch Ophthalmol 118:615-621
7. Drummond MF, O'Brien BJ, Stoddart GL, Torrance GW (1997) Methods for the economic evaluation of health care programmes, 2nd edn. Oxford University Press, Oxford, pp 139-199

8. Stein JD (2004) Disparities between ophthalmologists and their patients in estimating quality of life. Curr Opin Ophthalmol 5:238-243

9. Brown GC, Brown MM, Sharma S, Busbee B, Landy J (2002) A cost-utility analysis of interventions for severe proliferative vitreoretinopathy. Am J Ophthalmol 133:365-372

10. Schiffman RM, Walt JG, Jacobsen G, Doyle JJ, Lebovics G, Sumner W (2003) Utility assessment among patients with dry eye disease. Ophthalmology 110:1412-1419

11. Buchholz P, Steeds CS, Stern LS, Wiederkehr DP, Doyle JJ, Katz LM, Figueiredo FC (2006) Utility assessment to measure the impact of dry eye disease. Ocul Surf. 4(3):155-161

12. Scott IU, Smiddy WE, Feuer W, Merikansky A (1998) Vitreoretinal surgery outcomes Results of a subject satisfaction/functional status survey. Ophthalmology 105:795-803

13. Walt J (2004) Ocular Surface Disease Index (OSDI) administration and scoring manual. Allergan Inc, Irvine

14. Brown MM, Brown GC, Sharma S, Smith AF, Landy J (2001) A utility analysis correlation with visual acuity: methodologies and vision in the better and poorer eyes. Int Ophthalmol 24(3):123-127

15. Movahedan A, Djalilian AR (2012) Cataract surgery in the face of ocular surface disease. Curr Opin Ophthalmol 23(1):68-72. https://doi.org/10.1097/ICU.0b013e32834d 90b7.Review PubMed PMID: 22081034

16. Ferguson BA, Buxton MJ, Drummond MF (1990) Measuring and valuing health states related to visual impairment: a review of the literature, concepts, and methods. In: Drummond MF (ed.) Measuring the quality of life of people with visual impairment: proceedings of a workshop, Washington, DC: US Dept of Health and Human Services; 1990, pp 69-92. National Institutes of Health Publication 903078

17. Rosen PN, Kaplan RM, David K (2005) Measuring outcomes of cataract surgery using the Quality of Well-Being Scale and VF-14 Visual Function Index. J Cataract Refract Surg 31:369-378

18. Chan CW, Wong JC, Chan KS, Wong WK, Tam KC, Chau PS (2003) Evaluation of quality of life in patients with cataract in Hong Kong. J Cataract Refract Surg 29:1753-1760

19. Gothwal VK, Wright TA, Lamoureux EL, Pesudovs K (2010) Measuring outcomes of cataract surgery using the Visual Function Index-14. J Cataract Refract Surg 36:1181-1188

20. Lin IC, Wang IJ, Lei MS, Lin LL, Hu FR (2008) Improvements in vision-related quality of life with AcrySof IQ SN60WF aspherical intraocular lenses. J Cataract Refract Surg 34:1312-1317

21. Zhang F, Sugar A, Jacobsen G, Collins M (2011) Visual function and spectacle independence after cataract surgery: bilateral diffractive multifocal intraocular lenses versus monovision pseudophakia. J Cataract Refract Surg $37: 853-858$ 
22. Khanal S, Tomlinson A, Esakowitz L et al (2008) Changes in corneal sensitivity and tear physiology after phacoemulsification. Ophthalmic Physiol Opt 28(2):127-134

23. Brazier J, Roberts J, Tsuchiya A, Busschbach J (2004) A comparison of the EQ-5D and SF-6D across seven patient groups. Health Econ 13:873-884

24. Stavem K, Frøland SS, Hellum KB (2005) Comparison of preference-based utilities of the 15D, EQ-5D and SF-6D in patients with HIV/AIDS. Qual Life Res 14:971-980
25. Marra CA, Woolcott JC, Kopec JA et al (2005) A comparison of generic, indirect utility measures (the HUI2, HUI3, SF-6D, and the EQ-5D) and disease-specific instruments (the RAQoL and the HAQ) in rheumatoid arthritis. Soc Sci Med 60:1571-1582

26. WHO (1993) The development of the WHO quality of life assessment instrument. World Health Organization, Geneva

27. Ferreira PL, Ferreira LN, Pereira LN (2008) How consistent are health utility values? Qual Life Res 17:1031-1042 